ARTICLE

DOI: $10.1038 / s 41467-018-06244-8$

\title{
Nanomaterials design for super-degenerate electronic state beyond the limit of geometrical symmetry
}

\author{
Naoki Haruta (1) 1,2, Takamasa Tsukamoto (1) 1,2, Akiyoshi Kuzume ${ }^{1,2}$, Tetsuya Kambe (iD ${ }^{1,2}$ \& \\ Kimihisa Yamamoto ${ }^{1,2}$
}

Spherical atoms have the highest geometrical symmetry. Due to this symmetry, atomic orbitals are highly degenerate, leading to closed-shell stability and magnetism. No substances with greater degrees of degeneracy are known, due to geometrical limitations. We now propose that realistic magnesium, zinc, and cadmium clusters having a specific tetrahedral framework possess anomalous higher-fold degeneracies than spherical symmetry. Combining density functional theory calculations with simple tight-binding models, we demonstrate that these degeneracies can be attributed to dynamical symmetry. The degeneracy condition is fully identified as an elegant mathematical sequence involving interatomic parameters. The introduction of dynamical symmetry will lead to the discovery of a novel category of substances with super-degenerate orbitals.

\footnotetext{
${ }^{1}$ Institute of Innovative Research, Tokyo Institute of Technology, Yokohama 226-8503, Japan. ${ }^{2}$ ERATO, JST, Kawaguchi, Saitama 332-0012, Japan. These authors contributed equally: Naoki Haruta, Takamasa Tsukamoto. Correspondence and requests for materials should be addressed to

K.Y. (email: yamamoto@res.titech.ac.jp)
} 
$\mathrm{S}$ ymmetry is the most fundamental concept in both physics and chemistry ${ }^{1-3}$, and the basic properties of classical and quantum systems can be derived based on this concept. In real space, atoms have the highest geometrical symmetry, threedimensional spherical symmetry $O(3)$. As a result of this symmetry, these species possess atomic orbitals with the high degrees of degeneracy, such as $d$ orbitals, which have a fivefold degeneracy. This degeneracy in turn leads to certain properties, such as closed-shell stability and magnetism. However, species having higher degrees of degeneracy than atoms have not yet been known, due to the limitations of geometrical symmetry. It is of interest to consider whether or not it is possible to overcome this limitation.

Recently, the concept of a superatom has been proposed and developed $^{4-8}$. A superatom is analogous to an atom but with a higher-order structure: highly symmetrical metal clusters possess delocalized molecular orbitals, the shapes of which are just like those of atomic orbitals. This analogy can be understood based on the three-dimensional spherical jellium $\operatorname{model}^{9}$. As the full occupation of superatomic orbitals results in stable closed shells, the numbers of valence electrons necessary for closed shells are termed magic numbers. Clusters satisfying the magic number requirement have been detected and characterized by gas-phase spectroscopic methods. The most outstanding example is an aluminum cluster, $\mathrm{Al}_{13}{ }^{-}$, having $I_{\mathrm{h}}$ symmetry ${ }^{4}$. The molecular orbitals of this cluster are superatomic $S, P, D, F, \cdots$ orbitals and a stable closed-shell structure is formed similar to that of a halogen anion. Various other examples have also been reported, including $\mathrm{Au}_{20}$ having $T_{\mathrm{d}}$ symmetry ${ }^{10}$. The spherical jellium model has been applied with considerable success in cluster science. However, even if a cluster belongs to the highest point group, $I_{\mathrm{h}}$, its superatomic orbitals will split depending on their irreducible representations. The degree of degeneracy must be less than six based on point-group theory ${ }^{11}$, with the exception of spin-orbital degeneracy.

Non-geometrical symmetry has also been known, which gives rise to a greater degree of orbital degeneracy than that of spherical symmetry $O(3)^{3,12}$. As this symmetry originates not from geometrical properties but rather from dynamical characteristics, it is referred to as dynamical symmetry. A typical example is the hydrogen atom, in which the unoccupied atomic orbitals are highly degenerate, because its Hamiltonian possesses symmetry associated with the Laplace-Runge-Lenz vector ${ }^{13}$, in addition to three-dimensional spherical symmetry. As a result, the atom formally possesses four-dimensional spherical symmetry. Another example is the three-dimensional isotropic harmonic oscillator, in which the $2 S$ and $1 D$ orbitals are 6 -fold degenerate, the $2 P$ and $1 F$ orbitals are 10 -fold degenerate, and the $3 S, 2 D$, and $1 G$ orbitals are 15 -fold degenerate, and so on. This occurs because the Hamiltonian of this oscillator has $U(3)$ symmetry $1,3,12,14$. With the exception of the extreme example of the hydrogen atom, such dynamical symmetry has not yet been found in an actual substance. However, it is expected that species with this type of symmetry could exceed the spherical symmetry of atoms and exhibit unique electronic and magnetic properties.

Here we demonstrate that realistic magnesium, zinc, and cadmium clusters having a specific tetrahedral framework possess anomalous higher-fold degeneracies than spherical symmetry from first principles. In addition, by means of simple tight-binding models and group-theoretical analyses, we elucidate that these degeneracies can be attributed to dynamical symmetry.

\section{Results}

First-principles calculations. Density functional theory (DFT) calculations were performed for $T_{\mathrm{d}}$ symmetrical structures with valence electron numbers sufficient for the full occupation of superatomic orbitals. Figure $1 \mathrm{a}, \mathrm{b}$ show the molecular orbital levels obtained for $\mathrm{Zn}_{4}, \mathrm{Zn}_{10}, \mathrm{Zn}_{20}$, and $\mathrm{Zn}_{35}$. Owing to their high geometrical symmetry, these orbitals can be ascribed to superatomic $S, P, D, F, \cdots$ orbitals, depending on the orbital angular momenta (Fig. 1c). A remarkable aspect of these results is that the occupied orbitals have unusual higher-fold degeneracies: the $2 S$ and $1 D$ orbitals are 6 -fold degenerate, the $2 P$ and $1 F$ orbitals are 10 -fold degenerate, and the $3 S, 2 D$, and $1 G$ orbitals are 15 -fold degenerate. Such degeneracies are usually impossible, even in a spherical system. It should be noted that this degeneracy pattern is also consistent with that of the three-dimensional isotropic harmonic oscillator (Supplementary Fig. 1), as discussed further on. Equivalent degrees of degeneracy were also found in the case of other similar clusters, $X_{4}, X_{10}, X_{20}$, and $X_{35}(X=\mathrm{Mg}, \mathrm{Cd})$, as shown in Supplementary Figs. 2 and 3. Prior theoretical studies of $\mathrm{Mg}, \mathrm{Zn}$, and Cd clusters have noted the closed-shell structures of these species ${ }^{15-21}$. These neutral clusters have never been experimentally detected due to the associated technical difficulties, although their charged clusters having similar atomicity have been reported ${ }^{2-29}$. Figure $1 \mathrm{~d}$ plots the $2 S\left(a_{1}\right), 1 D\left(t_{2}\right)$, and $1 D(e)$ molecular orbital levels of $\mathrm{Mg}_{10}, \mathrm{Zn}_{10}, \mathrm{Cd}_{10}, \mathrm{Si}_{10}, \mathrm{Ge}_{10}, \mathrm{Sn}_{10}$, and $\mathrm{Pb}_{10}$ (see also Supplementary Figs. 4 and 5). Although all the clusters have $T_{\mathrm{d}}$ symmetry, the degrees of level splitting are different. $\mathrm{Mg}_{10}, \mathrm{Zn}_{10}$, and $\mathrm{Cd}_{10}$ have higher-fold orbital degeneracies, whereas $\mathrm{Si}_{10}, \mathrm{Ge}_{10}, \mathrm{Sn}_{10}$, and $\mathrm{Pb}_{10}$ have greater degrees of level splitting. The reason for this difference is discussed further on. In addition to these homonuclear clusters, several heteronuclear clusters with higher-fold degeneracies were also found: $\mathrm{Zn}_{6} \mathrm{Cd}_{4}$ and $\mathrm{Cd}_{6} \mathrm{Zn}_{4}$ (Supplementary Fig. 6). In particular, $\mathrm{Cd}_{6} \mathrm{Zn}_{4}$ exhibits an extremely small energy difference of only $15 \mathrm{meV}$ between its $1 D$ and $2 S$ orbitals (cf. $80 \mathrm{meV}$ for $\mathrm{Zn}_{10}$ ). For comparison purposes, other heteronuclear clusters, including $\mathrm{Al}_{6} \mathrm{Sn}_{4}, \mathrm{Ga}_{6} \mathrm{Sn}_{4}$, and $\mathrm{In}_{6} \mathrm{Sn}_{4}$, are also shown in Supplementary Figs. 7 and 8 . Among these, $\mathrm{Al}_{6} \mathrm{Sn}_{4}$ exhibits significant splitting of the $1 D$ and $2 S$ orbitals $(1.218 \mathrm{eV})$.

Tight-binding model analyses. To interpret these results theoretically, simple tight-binding models ${ }^{2,30,31}$ were constructed and analyzed as follows (Fig. 2). For simplicity, only s-type valence atomic orbitals were taken into account. First, a tight-binding model Hamiltonian for a four-atom tetrahedral structure was constructed as

$$
H_{4}=\left(\begin{array}{cccc}
\varepsilon & t & t & t \\
t & \varepsilon & t & t \\
t & t & \varepsilon & t \\
t & t & t & \varepsilon
\end{array}\right)
$$

where $\varepsilon$ is an atomic orbital energy and $t$ is a through-bond transfer integral (Fig. 2) ${ }^{32}$. The eigenvalues of $H_{4}$ can be analytically obtained as $\varepsilon+3 t, \varepsilon-t, \varepsilon-t, \varepsilon-t$, corresponding to the $1 S\left(a_{1}\right)$ and $1 P\left(t_{1}\right)$ levels, respectively. A threefold degeneracy associated with the $1 P$ levels appears, which is trivial within point-group theory. Second, a tight-binding model Hamiltonian 


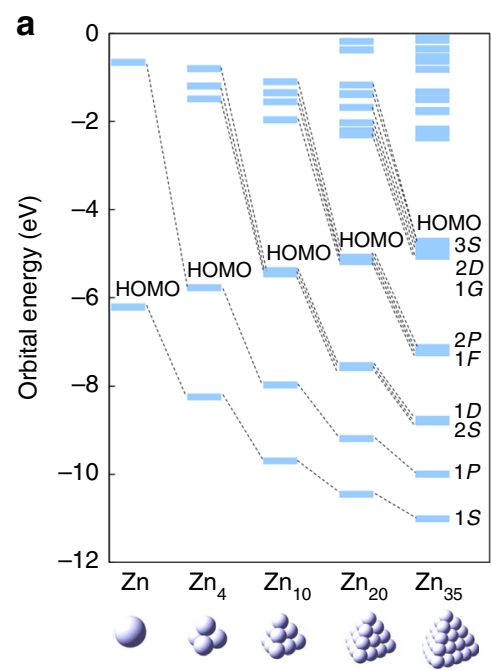

C

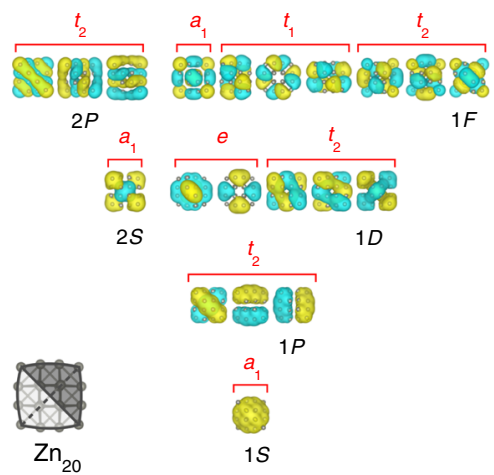

b

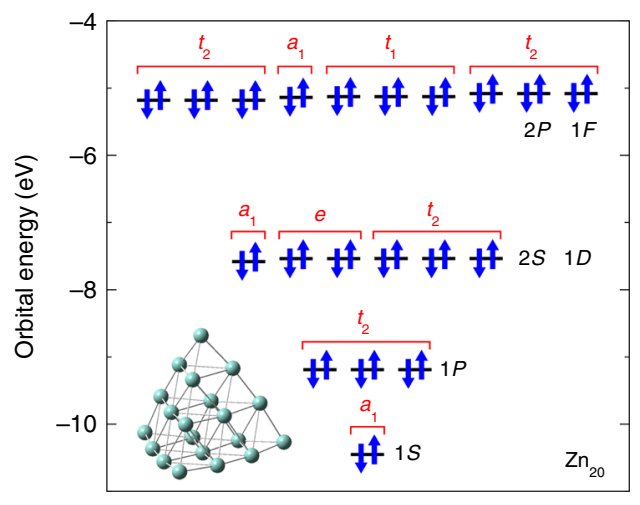

d

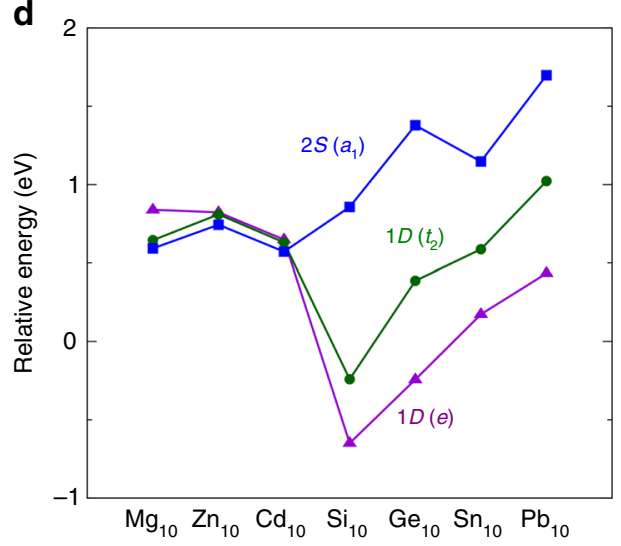

Fig. 1 DFT calculations of tetrahedral clusters. a Molecular orbital levels of $Z n_{1}, Z_{4}, Z n_{10}, Z n_{20}$, and $Z n_{35}$. $Z n_{35}$ favors a lower symmetry but was constrained to have $T_{d}$ symmetry for comparison purposes. b Occupied molecular orbital levels of $Z n_{20}$ with their irreducible representations and the optimized geometry. c Molecular orbitals of $\mathrm{Zn}_{20}$, the isosurface values of which are $1.0 \times 10^{-2}$ a.u. $\mathbf{d} 2 \mathrm{~S}$ and $1 \mathrm{D}$ molecular orbital levels of $\mathrm{Mg}_{10}$, $\mathrm{Zn}_{10}$, $\mathrm{Cd} \mathrm{d}_{10}$, $\mathrm{Si}_{10}, \mathrm{Ge}_{10}, \mathrm{Sn}_{10}$, and $\mathrm{Pb}_{10}$. The molecular orbital levels of each cluster have been shifted such that they originate at the atomic orbital level of the constitutive element. All the above calculations were carried out at the B3LYP/LanL2DZ level of theory implemented in Gaussian 09, Rev. E.0139

for a ten-atom tetrahedral structure was constructed as

$$
H_{10}=\left(\begin{array}{cccccccccc}
\varepsilon & t_{2} & t_{2} & t_{2} & 0 & 0 & 0 & 0 & 0 & 0 \\
t_{2} & \varepsilon & t_{1} & t_{1} & t_{2} & t_{1} & t_{1} & 0 & 0 & 0 \\
t_{2} & t_{1} & \varepsilon & t_{1} & 0 & t_{1} & 0 & t_{2} & t_{1} & 0 \\
t_{2} & t_{1} & t_{1} & \varepsilon & 0 & 0 & t_{1} & 0 & t_{1} & t_{2} \\
0 & t_{2} & 0 & 0 & \varepsilon & t_{2} & t_{2} & 0 & 0 & 0 \\
0 & t_{1} & t_{1} & 0 & t_{2} & \varepsilon & t_{1} & t_{2} & t_{1} & 0 \\
0 & t_{1} & 0 & t_{1} & t_{2} & t_{1} & \varepsilon & 0 & t_{1} & t_{2} \\
0 & 0 & t_{2} & 0 & 0 & t_{2} & 0 & \varepsilon & t_{2} & 0 \\
0 & 0 & t_{1} & t_{1} & 0 & t_{1} & t_{1} & t_{2} & \varepsilon & t_{2} \\
0 & 0 & 0 & t_{2} & 0 & 0 & t_{2} & 0 & t_{2} & \varepsilon
\end{array}\right)
$$

where $t_{1}$ and $t_{2}$ are transfer integrals corresponding to different types of bonds (Fig. 2). It should be noted that the absolute value of a transfer integral increases as an interatomic distance decreases (Supplementary Fig. 9). The analytical eigenvalues were obtained as $\varepsilon+2 t_{1}-\sqrt{4 t_{1}^{2}+6 t_{2}^{2}}, \varepsilon+\sqrt{2} t_{2}, \varepsilon+\sqrt{2} t_{2}, \varepsilon+\sqrt{2} t_{2}$, $\varepsilon+2 t_{1}+\sqrt{4 t_{1}^{2}+6 t_{2}^{2}}, \varepsilon-\sqrt{2} t_{2}, \varepsilon-\sqrt{2} t_{2}, \varepsilon-\sqrt{2} t_{2}, \varepsilon-2 t_{1}, \varepsilon-$ $2 t_{1}$, corresponding to the $1 S\left(a_{1}\right), 1 P\left(t_{1}\right), 2 S\left(a_{1}\right), 1 D\left(t_{2}\right)$, and $1 D(e)$ levels, respectively. As illustrated in Fig. 3a, an unexpected degeneracy point associated with $1 D\left(t_{2}\right), 1 D(e)$, and $2 S\left(a_{1}\right)$ appears. The condition producing this degeneracy was found not to be a regular tetrahedron $\left(t_{1}=t_{2}\right)$ but rather an inflated tetrahedron $\left(t_{2}=\sqrt{2} t_{1}\right)$, and this simple $t_{2}$-to- $t_{1}$ ratio implies the existence of hidden symmetry. However, this abnormal degeneracy cannot be explained, at least within point-group theory, because transfer integral ratios corresponding to non-equivalent bonds are outside the scope of the theory.

The validity of the simple ten-atom system model was subsequently examined. Table 1 lists the model parameters (that is, $t_{1}$ and $t_{2}$ ) obtained from the DFT calculations. These values were estimated as half the level of splitting between bonding and anti-bonding orbitals of $s$-type valence electrons. The energy difference, $\Delta$, between the $2 S$ and $1 D$ molecular orbitals can be calculated from the simple tight-binding model $\left(\Delta=-4 t_{1}-\right.$ $\sqrt{4 t_{1}^{2}+6 t_{2}^{2}}$ ). In addition, $\Delta$ can also be determined directly from DFT calculations (Table 1). The validity of the present model can be ascertained by comparing these values. Figure $3 \mathrm{~b}$ plots the degree of level splitting, $\Delta / t_{1}$, as a function of the transfer integral ratio, $t_{2} / t_{1}$. It is evident that the DFT-based values (blue squares) perfectly follow the simple model curve. Thus, the present tightbinding model employing solely through-bond transfer integrals 
is in very good agreement with the DFT calculations. This agreement is attributed to the present system being constructed of three-simplexes, or four-atom tetrahedrons, which has few through-space interactions ${ }^{32}$ (see also Supplementary Note 1). Remarkably, $\mathrm{Mg}_{10}, \mathrm{Zn}_{10}$, and $\mathrm{Cd}_{10}$ are all located very close to an ideal degeneracy point $\left(t_{2}=\sqrt{2} t_{1}\right)$, in contrast to $\mathrm{Si}_{10}, \mathrm{Ge}_{10}, \mathrm{Sn}_{10}$, and $\mathrm{Pb}_{10}$. This result explains why the degrees of level splitting vary between different elements.

In the same manner, tight-binding model Hamiltonians for larger systems were also constructed (Supplementary Notes 2 and 3; Supplementary Figs. 10 and 11). A general condition for the anomalous orbital degeneracy was subsequently fully identified, as shown in Fig. 4a. Surprisingly, the degeneracy condition can be represented as an elegant square-root mathematical sequence (appearing in the so-called spiral of Theodorus) involving the ratio of transfer integrals. In each case, the degeneracy condition corresponds not to a regular tetrahedron but rather to an inflated tetrahedron.

\section{Discussion}

To elucidate the cause of the higher-fold degeneracies, the symmetry of each model was analyzed. The Hamiltonian, $H_{N}$ $(N=1,4,10,20, \cdots)$, satisfying the degeneracy condition can be rewritten as

$$
H_{N}=\{\varepsilon+3(n-1) t\} I-4 t \sum_{i=1}^{3} a_{i}^{\dagger} a_{i}
$$
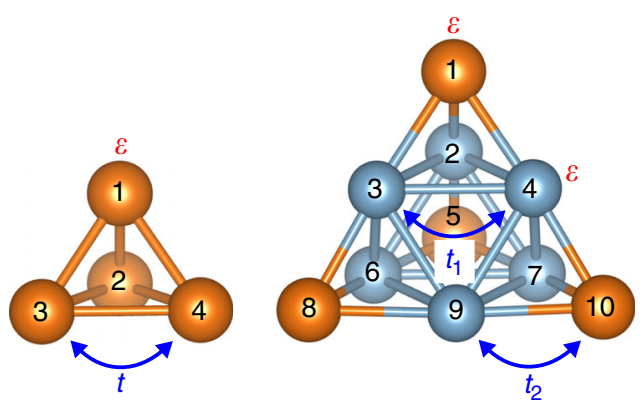

Fig. 2 Model parameters for four-atom and ten-atom systems where $n$ is the number of constitutive layers in the $N$-atom tetrahedral system, $I$ is the identity matrix, and $a_{i}^{\dagger}$ and $a_{i}(i=1,2,3)$ are creation and annihilation operators on $N$-dimensional Hilbert space. Thus, the present system is equivalent to the harmonic oscillator with $U(3)$ symmetry under the degeneracy condition. In fact, $H_{\mathrm{N}}$ is invariant under the following transformations,

$$
a_{i} \mapsto \sum_{j=1}^{3} u_{i j} a_{j}, \quad a_{i}^{\dagger} \mapsto \sum_{k=1}^{3} u_{i k}^{*} a_{k}^{\dagger} \quad(i=1,2,3),
$$

where the unitary condition is satisfied as

$$
\sum_{i=1}^{3} u_{i k}^{*} u_{i j}=\delta_{k j}
$$

The energy spectra obtained under the degeneracy conditions are equivalent to that of the harmonic oscillator, as shown in Fig. $4 \mathrm{~b}$. This equality is believed to result from the coincidence of atomicity in the $n$th layer of the tetrahedron and the orbital degeneracy of the $n$th energy of the harmonic oscillator (Supplementary Note 4; Supplementary Figs. 12 and 13). The group chain $U(3) \supset O(3)$ indicates that the present system has a higher degree of symmetry than the highest geometrical symmetry.

In general, all metal clusters cannot have $O(3)$, because they have finite numbers of constitutive atoms in their geometrical structures. Therefore, it is just an approximative picture that some magic clusters satisfy $O(3)$. This is the shell model ${ }^{9}$, in which geometrical information is all abstracted and instead a spherical structure is assumed for simplicity. In contrast to such a traditional view, the present work demonstrates that tetrahedral clusters are able to satisfy the dynamical symmetry higher than $O$ (3) exactly. This is notable because geometrical abstraction is not necessary any more. Figure 3 a illustrates that the energy of $1 D\left(t_{2}\right)$ is closer to that of $2 S\left(a_{1}\right)$ than to that of $1 D(e)$, because the $1 D\left(t_{2}\right)$ and $2 S\left(a_{1}\right)$ orbitals have distributions on the four vertex atoms, whereas the $1 D(e)$ orbitals have no distribution at the vertices (see Supplementary Fig. 14). This is the intrinsic orbital splitting pattern of tetrahedral clusters. As a result, the degeneracy of $1 D$ $\left(t_{2}\right)$ and $1 D(e)$ does not appear independently of $2 S\left(a_{1}\right)$. Instead, $1 D\left(t_{2}\right), 1 D(e)$, and $2 S\left(a_{1}\right)$ are all degenerate just at a triple point. This implies that tetrahedral clusters achieve the dynamical symmetry not via $O(3)$. On the other hand, in the case of tetrahedral clusters being too inflated and quasi-spherical, $1 D\left(t_{2}\right)$ pairs with $1 D(e)$ rather than with $2 S\left(a_{1}\right)$, as shown in Fig. $1 \mathrm{~d}$.
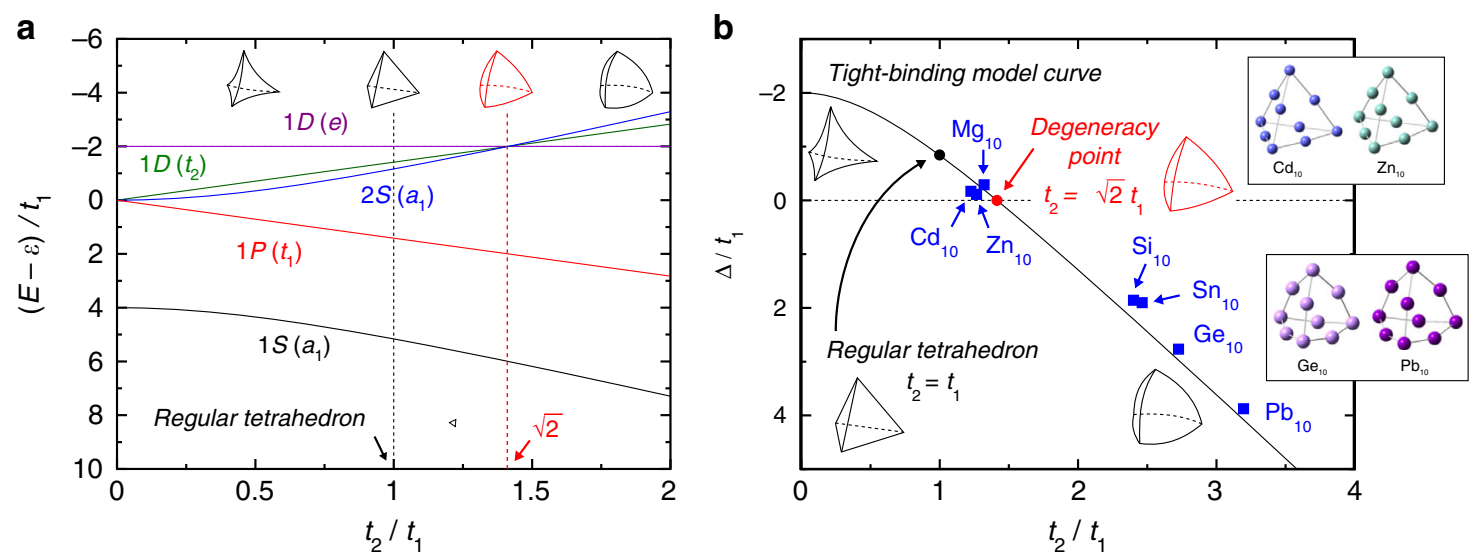

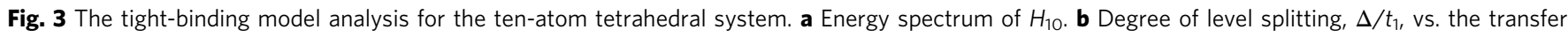
integral ratio, $t_{2} / t_{1}$. Blue squares indicate the DFT value of each level splitting. All the parameters were determined based on DFT calculations at the B3LYP/LanL2DZ level of theory (Table 1). DFT-based optimized structures are also included 
Table 1 The evaluated parameters by DFT calculations

\begin{tabular}{lllllll} 
& $\mathbf{M g}_{\mathbf{1 0}}$ & $\mathbf{Z n}_{\mathbf{1 0}}$ & $\mathbf{C d}_{\mathbf{1 0}}$ & $\mathbf{S i}_{\mathbf{1 0}}$ & $\mathbf{G e}_{\mathbf{1 0}}$ & $\mathbf{S n}_{\mathbf{1 0}}$ \\
\hline$t_{1}(\mathrm{eV})$ & -0.85 & -0.72 & -0.46 & -0.81 & -0.59 & -0.51 \\
$t_{2}(\mathrm{eV})$ & -1.12 & -0.91 & -0.56 & -1.95 & -1.60 & -1.26 \\
$\Delta_{\text {TB }}(\mathrm{eV})$ & 0.17 & 0.22 & 0.18 & -1.79 & -1.74 & -1.04 \\
$\Delta_{\text {DFT }}(\mathrm{eV})$ & 0.25 & 0.08 & 0.08 & -1.51 & -1.62 & -1.21 \\
\hline
\end{tabular}

$\Delta$ denotes the $1 D(e)$ energy minus that of $2 S\left(a_{1}\right) . \Delta_{\mathrm{TB}}$ and $\Delta_{\mathrm{DFT}}$ were evaluated by the tight-binding model $\left(-4 t_{1}-\sqrt{4 t_{1}^{2}+6 t_{2}^{2}}\right)$ and directly by DFT calculations, respectively. The B3LYP/LanL2DZ level of theory was employed for the DFT calculations

a
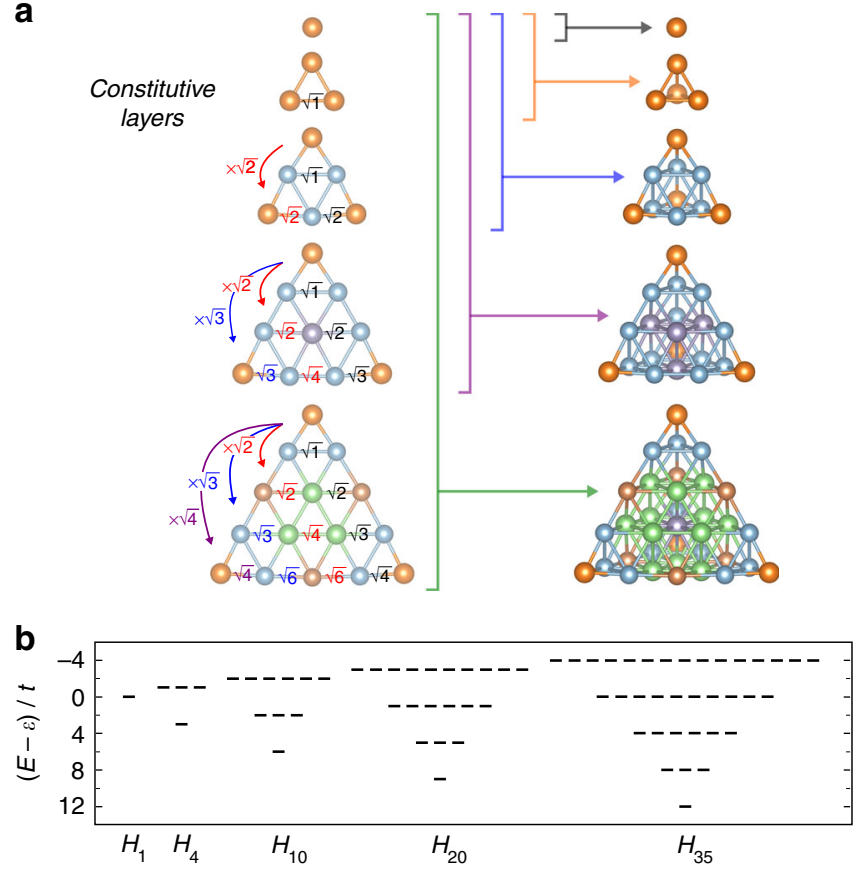

Fig. 4 The tight-binding model analyses for tetrahedral clusters with various sizes. a Ratios of transfer integrals giving rise to the anomalous degeneracy, in which a square-root mathematical sequence appears. $\mathbf{b}$ Energy spectra of the tight-binding models under the degeneracy conditions. Here, $t$ denotes a transfer integral whose absolute value is the smallest in each model

A prior theoretical study has reported that the spherical jellium model spontaneously deforms in a tetrahedral direction, if its shape is relaxed under the condition that the total number of valence electrons coincides with the magic number of the three-dimensional isotropic harmonic oscillator ${ }^{15}$. The present study illustrates the reason why tetrahedrally deformed orbitals are special by identifying the exact $U(3)$ point in the tetrahedral frameworks on the basis of the simple tight-binding models.

In conclusion, nanomaterials that surpass the symmetry of spherical atoms can be realized by considering not only geometrical symmetry but also dynamical symmetry. Owing to this nontrivial symmetry, these species have super-degenerate molecular orbitals that give rise to an extremely high discrete density of states around the Fermi level. Carrier doping into these superdegenerate orbitals could lead to excellent electric conductivity, as long as the unfavorable Jahn-Teller effect is not so considerable. In addition, the spin arrangement in super-degenerate orbitals could yield unique magnetism. Unlike atoms, it is impossible to realize arbitrary spin states, because of the Jahn-Teller effect. However, a certain high spin state can be stabilized by matching the number of singly-occupying electrons with the degree of super-degeneracy through alloying or changing a constitutive element (see Supplementary Figs. 15 and 16). Such a state could be obtained by synthesizing clusters under a magnetic field. Thus, the control of dynamical symmetry should lead to the development of next-generation electronic and magnetic materials.

The proposed clusters are to be viable ${ }^{33}$ in light of prior experimental studies. The laser vaporization techniques combined with time-of-flight mass spectrometry produced $\mathrm{Mg}, \mathrm{Zn}, \mathrm{Cd}$ clusters with tetrahedral atomicities in the gas phase ${ }^{22-29}$. A softlanding method onto self-assembled monolayers should be effective to obtain the clusters as materials ${ }^{34}$. As for the liquid phase, a template-based method can be used to fabricate the clusters $^{35}$. Ligand protection might realize the isolation and crystallization ${ }^{36}$. It is also helpful to refer to the crystallization of Zintl clusters ${ }^{37}$. Furthermore, much attention should be paid to emerging cocrystallization techniques of superatomic clusters and fullerenes ${ }^{38}$. Some of these synthetic methods should be suitable for the proposed clusters. The present study features $\mathrm{Mg}, \mathrm{Zn}$, and $\mathrm{Cd}$ tetrahedral clusters. However, other tetrahedral clusters with inappropriate structures for super-degeneracy, being too inflated, are also of interest under mechanical pressure.

\section{Methods}

First-principles calculations. By employing the DFT method, geometry optimizations and vibrational analyses were performed for $T_{\mathrm{d}}$ symmetrical structures with valence electron numbers sufficient for the full occupation of superatomic orbitals. Specifically, the homonuclear clusters $X_{4}, X_{10}, X_{20}$, and $X_{35}(X=\mathrm{Mg}, \mathrm{Zn}$, $\mathrm{Cd})$ and the heteronuclear clusters $X_{6} Y_{4}(X, Y=\mathrm{Zn}, \mathrm{Cd})$ were calculated. These clusters have 8, 20, 40, 70, and $20 s$-type valence electrons, respectively. For comparison purposes, the 40 valence electron systems $\mathrm{Si}_{10}, \mathrm{Ge}_{10}, \mathrm{Sn}_{10}, \mathrm{~Pb}_{10}, \mathrm{Al}_{6} \mathrm{Sn}_{4}$ $\mathrm{Ga}_{6} \mathrm{Sn}_{4}$, and $\mathrm{In}_{6} \mathrm{Sn}_{4}$, the 14 valence electron system $\mathrm{Au}_{6} \mathrm{Zn}_{4}$, and the 30 valence electron system $\mathrm{Tl}_{10}$ were also calculated. All the DFT calculations were conducted with the B3LYP functional and LanL2DZ basis set, using the Gaussian 09, Rev. E.01 program package ${ }^{39}$. All the structure data are available in Supplementary Tables 1-23.

\section{Data availability}

The data that support the findings of this study are available from the corresponding author upon reasonable request.

Received: 7 May 2018 Accepted: 24 August 2018

Published online: 14 September 2018

\section{References}

1. Georgi, H. Lie Algebras in Particle Physics: From Isospin to Unified Theories. (Westview Press, Boulder, 1999).

2. Ceulemans, A. J. Group Theory Applied to Chemistry. Theoretical Chemistry and Computational Modelling. (Springer, Dordrecht, 2013).

3. Thyssen, P. \& Ceulemans, A. J. Shattered Symmetry: Group Theory from the Eightfold Way to the Periodic Table. (Oxford University Press, Oxford, 2017).

4. Bergeron, D. E., Castleman, A. W. Jr., Morisato, T. \& Khanna, S. N. Formation of $\mathrm{Al}_{13} \mathrm{I}^{-}$: Evidence for the superhalogen character of $\mathrm{Al}_{13}$. Science 304, 84-87 (2004).

5. Reber, A. C., Khanna, S. N. \& Castleman, A. W. Jr. Superatom compounds, clusters, and assemblies: Ultra alkali motifs and architectures. J. Am. Chem. Soc. 129, 10189-10194 (2007).

6. Castleman, A. W. Jr. \& Khanna, S. N. Clusters, superatoms, and building blocks of new materials. J. Phys. Chem. C 113, 2664-2675 (2009). 
7. Claridge, S. A. et al. Cluster-assembled materials. ACS Nano 3, 244-255 (2009).

8. Tomalia, D. A. \& Khanna, S. N. A systematic framework and nanoperiodic concept for unifying nanoscience: Hard/soft nanoelements, superatoms, metaatoms, new emerging properties, periodic property patterns, and predictive Mendeleev-like nanoperiodic tables. Chem. Rev. 116, 2705-2774 (2016).

9. de Heer, W. A. The physics of simple metal clusters: Experimental aspects and simple models. Rev. Mod. Phys. 65, 611-676 (1993).

10. Li, J., Li, X., Zhai, H.-J. \& Wang, L.-S. Au $\mathrm{Au}_{20}$ : A tetrahedral cluster. Science 299, 864-867 (2003).

11. Altmann, S. L. \& Herzig, P. Point-Group Theory Tables. 2nd edn, (Clarendon Press, Wien, 2011)

12. Weinberg, S. Lectures on Quantum Mechanics. (Cambridge University Press, Cambridge, 2015).

13. Pauli, W. On the spectrum of the hydrogen from the standpoint of the new quantum mechanics. Z. Phys 36, 336-363 (1926).

14. Fradkin, D. M. Three-dimensional isotropic harmonic oscillator and $\mathrm{SU}_{3}$. Am. J. Phys. 33, 207-211 (1965).

15. Reimann, S. M., Koskinen, M., Häkkinen, H., Lindelof, P. E. \& Manninen, M. Magic triangular and tetrahedral clusters. Phys. Rev. B 56, 12147-12150 (1997).

16. Zhao, J. Density-functional study of structures and electronic properties of $\mathrm{Cd}$ clusters. Phys. Rev. A. 64, 043204 (2001).

17. Serra, L., Reinhard, P.-G. \& Suraud, E. Density functional calculations for shell closures in Mg clusters. Eur. Phys. J. D 18, 327-333 (2002).

18. Wang, J., Wang, G. \& Zhao, J. Nonmetal-metal transition in $\mathrm{Zn}_{n}(n=2-20)$ clusters. Phys. Rev. A. 68, 013201 (2003).

19. Johansson, M. P. \& Pyykkö, P. The importance of being tetrahedral: The cadmium pyramids $\mathrm{Cd}_{N} ; N=4,10,20,35$ and 56. Phys. Chem. Chem. Phys. 6, 2907-2909 (2004).

20. Heidari, I., De, S., Ghazi, S. M., Goedecker, S. \& Kanhere, D. G. Growth and structural properties of $\mathrm{Mg}_{N}(N=10-56)$ clusters: Density functional theory study. J. Phys. Chem. A 115, 12307-12314 (2011).

21. Köhn, A., Weigend, F. \& Ahlrichs, R. Theoretical study on clusters of magnesium. Phys. Chem. Chem. Phys. 3, 711-719 (2001).

22. Katakuse, I. et al. Correlation between mass distributions of zinc, cadmium clusters and electronic shell structure. Int. J. Mass Spectrom. Ion Processes 69, 109-114 (1986).

23. Katakuse, I. et al. Mass distributions of positive and negative cluster ions of zinc and cadmium. Int. J. Mass Spectrom. Ion Processes 91, 85-91 (1989).

24. Diederich, T., Döppner, T., Braune, J., Tiggesbäumker, J. \& Meiwes-Broer, K.-H. Electron delocalization in magnesium clusters grown in supercold helium droplets. Phys. Rev. Lett. 86, 4807-4810 (2001).

25. Döppner, T., Diederich, T., Tiggesbäumker, J. \& Meiwes-Broer, K.-H. Femtosecond ionization of magnesium clusters grown in ultracold helium droplets. Eur. Phys. J. D 16, 13-16 (2001).

26. Thomas, O. C., Zheng, W., Xu, S. \& Bowen, K. H. Jr. Onset of metallic behavior in magnesium clusters. Phys. Rev. Lett. 89, 213403 (2002)

27. Diederich, T., Döppner, T., Fennel, T., Tiggesbäumker, J. \& Meiwes-Broer, K.-H. Shell structure of magnesium and other divalent metal clusters. Phys. Rev. A. 72, 023203 (2005).

28. Kostko, O., Wrigge, G., Cheshnovsky, O. \& v. Issendorff, B. Transition from a Bloch-Wilson to a free-electron density of states in $\mathrm{Zn}_{n}{ }^{-}$clusters. J. Chem. Phys. 123, 221102 (2005).

29. Kostko, O. Photoelectron spectroscopy of mass-selected sodium, coinage metal and divalent metal cluster anions. Ph.D. thesis (Albert-Ludwigs-Universität Freiburg, Freiburg im Breisgau, 2007).

30. Hosoya, H. Topological index. A newly proposed quantity characterizing the topological nature of structural isomers of saturated hydrocarbons. Bull. Chem. Soc. Jpn. 44, 2332-2339 (1971).

31. Ceulemans, A. J., Lijnen, E., Fowler, P. W., Mallion, R. B. \& Pisanski, T. Graph theory and the Jahn-Teller theorem. Proc. R. Soc. A 468, 971-989 (2012).
32. Hoffmann, R. Interaction of orbitals through space and through bonds. Acc. Chem. Res. 4, 1-9 (1971).

33. Hoffmann, R., von Ragué Schleyer, P. \& Schaefer, H. F. III Predicting molecules-More realism, please! Angew. Chem. Int. Ed. 47, 7164-7167 (2008).

34. Mitsui, M., Nagaoka, S., Matsumoto, T. \& Nakajima, A. Soft-landing isolation of vanadium-benzene sandwich clusters on a room-temperature substrate using $n$-alkanethiolate self-assembled monolayer matrixes. J. Phys. Chem. B 110, 2968-2971 (2006).

35. Crooks, R. M., Zhao, M., Sun, L., Chechik, V. \& Yeung, L. K. Dendrimerencapsulated metal nanoparticles: Synthesis, characterization, and applications to catalysis. Acc. Chem. Res. 34, 181-190 (2001).

36. Walter, M. et al. A unified view of ligand-protected gold clusters as superatom complexes. Proc. Natl. Acad. Sci. U. S. A. 105, 9157-9162 (2008).

37. Scharfe, S., Kraus, F., Stegmaier, S., Schier, A. \& Fässler, T. F. Zintl ions, cage compounds, and intermetalloid clusters of group 14 and group 15 elements. Angew. Chem. Int. Ed. 50, 3630-3670 (2011).

38. Pinkard, A., Champsaur, A. M. \& Roy, X. Molecular clusters: Nanoscale building blocks for solid-state materials. Acc. Chem. Res. 51, 919-929 (2018).

39. Frisch, M. J. et al. Gaussian 09 Revision E.01. (Gaussian Inc, Wallingford CT, 2015).

\section{Acknowledgements}

This study was supported by a grant from JST ERATO, Japan (number JPMJER1503).

\section{Author contributions}

N.H. and T.T. initiated and contributed equally to this work. K.Y. supervised it. N.H. and T.T. conducted the DFT calculations, constructed the tight-binding models, and analyzed the results of the calculations. N.H., T.T., A.K., T.K., and K.Y. participated in the discussion and the writing of the manuscript.

\section{Additional information}

Supplementary Information accompanies this paper at https://doi.org/10.1038/s41467018-06244-8.

Competing interests: The authors declare no competing interests.

Reprints and permission information is available online at http://npg.nature.com/ reprintsandpermissions/

Publisher's note: Springer Nature remains neutral with regard to jurisdictional claims in published maps and institutional affiliations.

Open Access This article is licensed under a Creative Commons Attribution 4.0 International License, which permits use, sharing, adaptation, distribution and reproduction in any medium or format, as long as you give appropriate credit to the original author(s) and the source, provide a link to the Creative Commons license, and indicate if changes were made. The images or other third party material in this article are included in the article's Creative Commons license, unless indicated otherwise in a credit line to the material. If material is not included in the article's Creative Commons license and your intended use is not permitted by statutory regulation or exceeds the permitted use, you will need to obtain permission directly from the copyright holder. To view a copy of this license, visit http://creativecommons.org/ licenses/by/4.0/

(C) The Author(s) 2018 